\title{
Prolonged survival in pancreatic cancer patients with increased regucalcin gene expression: Overexpression of regucalcin suppresses the proliferation in human pancreatic cancer MIA PaCa-2 cells in vitro
}

\author{
MASAYOSHI YAMAGUCHI ${ }^{1}$, SATORU OSUKA ${ }^{2}$, M. NEALE WEITZMANN ${ }^{3,4}$, \\ BASSEL F. EL-RAYES ${ }^{1}$, MAMORU SHOJI ${ }^{1}$ and TOMIYASU MURATA ${ }^{5}$
}

\begin{abstract}
Departments of ${ }^{1}$ Hematology and Medical Oncology, and ${ }^{2}$ Neurosurgery, Winship Cancer Institute, Emory University School of Medicine, Atlanta, GA 30322; ${ }^{3}$ Division of Endocrinology and Metabolism and Lipids, Department of Medicine, Emory University School of Medicine, 1329 WMRB, Atlanta, GA 30322; ${ }^{4}$ The Atlanta Department of Veterans Affairs Medical Center, Decatur, GA 30033, USA; ${ }^{5}$ Laboratory of Analytical Neurobiology, Faculty of Pharmacy, Meijo University, Yagotoyama 150, Tempaku, Nagoya 468-8503, Japan
\end{abstract}

Received December 13, 2015; Accepted January 15, 2016

DOI: 10.3892/ijo.2016.3409

\begin{abstract}
Approximately 90\% of all pancreatic cancers are pancreatic ductal adenocarcinomas (PDAC). PDAC is a highly aggressive malignancy and is one of the deadliest. This poor clinical outcome is due to the prominent resistance of pancreatic cancer to drug and radiation therapies. Regucalcin plays a pivotal role as a suppressor protein in signal transduction in various types of cells including tumor tissues. We demonstrated that the prolonged survival is induced in PDAC patients with increased regucalcin gene expression using a dataset of PDAC obtained from GEO database (GSE17891) together with the clinical annotation data file. Moreover, overexpression of regucalcin with full length was demonstrated to suppress the proliferation, cell death and migration in human pancreatic cancer MIA PaCa-2 (K-ras mutated) cells that possess resistance to drug and radiation therapies. Suppressive effects of regucalcin on cell proliferation and death were not seen in the cells overexpressed with regucalcin cDNA alternatively spliced variants (deleted exon 4 or deleted exon 4 and 5). Regucalcin was suggested to induce G1 and G2/M phase cell cycle arrest in MIA PaCa-2 cells. Suppressive effects of regucalcin on cell proliferation were independent of cell death. Overexpression of regucalcin was found to suppress signaling pathways including Akt, MAP kinase and SAPK/JNK, to increase the protein levels of $p 53$, a tumor suppresser, and to decrease K-ras, c-fos and c-jun, a oncogene, by suppressing
\end{abstract}

Correspondence to: Dr Masayoshi Yamaguchi, Department of Hematology and Medical Oncology, Winship Cancer Institute, Emory University School of Medicine, C-5054, 1365 C Clifton Road, NE, Atlanta, GA 30322, USA

E-mail: yamamasa1155@yahoo.co.jp

Key words: regucalcin, cell proliferation, cell death, migration, pancreatic cancer, MIA PaCa-2 cells signaling pathways that are related to signaling of K-ras. Regucalcin may play a potential role as a suppressor protein in human pancreatic cancer.

\section{Introduction}

Normal pancreas consists of two classes of cells: endocrine (hormone secreting) and exocrine (digestive enzyme producing). Depending on the cell of origin, pancreatic cancers can also be classified as endocrine or exocrine tumors. Approximately $90 \%$ of all pancreatic cancers are pancreatic ductal adenocarcinomas (PDAC), an exocrine pancreatic tumor that resembles the cells lining the pancreatic duct (1-3). Pancreatic cancer is a highly aggressive malignancy with a notoriously dismal prognosis, and currently available therapies are only minimally effective in treating this disease (4-6). Pancreatic cancer ranks fourth in cancer mortality and accounts for $<7 \%$ of all cancer-related death (1-3). This poor clinical outcome may be due to the prominent resistance to drugs and radiation therapies (4-7). The majority of pancreatic cancer has K-ras mutations with $90 \%$ possessing activating mutations in this oncogene (8-10). Oncogenic K-ras plays a critical role in much of the metabolic reprogramming seen in pancreatic cancer (1-4). The observed frequency of K-ras brings about the consistent expression of irregular Ras protein that causes aberrant activation of cell proliferation, migration, invasion and survival pathways $(1,10)$.

Regucalcin gene $(r g n)$ is localized on the $\mathrm{X}$ chromosome (11-13), and plays a pivotal role as a suppressor protein of multi-signaling pathway in various types of cells and tissues (14-16). The regucalcin gene expression is regulated by various hormonal factors including calcium-related process, calcium-regulating hormones, insulin, estrogen and other steroid hormones (17). Regucalcin is translocated from the cytoplasm to the nucleus in various types of cells (17). Regucalcin has been shown to play a role in the maintaining 
of intracellular calcium homeostasis and inhibiting various protein kinases, protein phosphatases and protein synthesis in the cytoplasm and nuclear DNA and RNA syntheses (14-17). Nuclear regucalcin has also been shown to regulate the gene expression of various proteins (17). Moreover, regucalcin has been found to suppress cell proliferation and apoptotic cell death that are mediated through various signaling factors $(19,20)$. Thus, regucalcin is proposed to play a cell physiologic role in maintaining cell homeostasis and function as a suppressor protein of intracellular signaling systems $(15,16)$.

Regucalcin has been shown to possess a pathophysiologic role in metabolic disorder and diseases. Noticeably, regucalcin has been shown to be involved in carcinogenesis (21). The regucalcin gene and its protein expressions were found to decrease in mammalian tumor tissues and human subjects in vivo (22). Regucalcin gene expression has been demonstrated to be downregulated in the development of carcinogenesis, suggesting a role of regucalcin as a suppressor protein in carcinogenesis. Moreover, overexpression of endogenous regucalcin was found to suppress the enhancement of cell proliferation in cloned rat hepatoma H4-II-E cells in vitro, of which regucalcin gene expression is suppressed $(19,23)$. Recently, we have demonstrated that exogenous regucalcin reveals suppressive effects on cell proliferation in human pancreatic cancer MIA $\mathrm{PaCa}-2$ (K-ras mutated) cells, which possess resistance to drugs and radiation therapies, in vitro (24).

The present study was undertaken to determine an involvement of regucalcin in human pancreatic cancer. We found prolonged survival in PDAC patients with the higher regucalcin gene expression using a dataset of PDAC obtained from GEO database (GSE17891) together with the clinical annotation data file, and that overexpression of endogenous regucalcin exhibits suppressive effects on the proliferation of MIA PaCa- 2 cells in vitro. These findings may support a potential role of regucalcin as a suppressor protein in human pancreatic cancer.

\section{Materials and methods}

Materials. Dulbecco's modified Eagle's medium (DMEM) with $4.5 \mathrm{~g} / 1$ glucose, L-glutamine and sodium pyruvate and antibiotics [(penicillin and streptomycin P/S)] were purchased from Corning (Mediatech, Inc. Manassas, VA, USA). Fetal bovine serum (FBS) was from HyClone Laboratories (Logan, UT, USA). Lipofectamine reagent was obtained from Promega (Madison, WI, USA). Tumor necrosis factor- $\alpha$ (TNF- $\alpha$ ) was from R\&D Systems (Minneapolis, MN, USA). Sodium butyrate, roscovitine, sulforaphane, PD98059, thapsigargin, Bay K8644, worthomannin, 5,6-dichloro-1- $\beta$ D-ribofuranosylbenzimidazole (DRB), caspase-3 inhibitor and all other reagents were purchased from Sigma-Aldrich (St. Louis, MO, USA) unless otherwise specified. Gemcitabine was obtained from Hospira, Inc. (Lake Forest, IL, USA). Gemcitabine and caspase-3 inhibitor were diluted in phosphate buffered saline (PBS) and other reagents were dissolved in $100 \%$ ethanol to use in the experiments.

Patient datasets. A dataset of 36 normal pancreas and 36 PDAC were obtained via the Gene Expression Omnibus (GEO) database (GSE15471) for analysis of regucalcin expres- sion (25). For outcome analysis, a dataset of 25 PDAC were also obtained from the GEO database (GSE17891) together with the clinical annotation data file (26). These datasets contained gene expression data derived from the Affymetrix U133_Plus2 platform. For microarray analysis, the expression and the raw expression data (CEL files) were summarized and normalized using the Robust Multi-array Average algorithm and the Bioconductor package affy (http://www. bioconductor.org/packages/2.0/bioc/html/affy.html).

The Spotfire DecisionSite for Functional Genomics software package (TIBCO Software, Palo Alto, CA, USA) was used for the visualization of microarray data. For protein expression analysis, a dataset of immunohistochemistry were obtained from the Human Protein Atlas (HPA) (www.proteinatlas. org), which is a database of proteins in human normal tissues and cancers $(27,28)$. We also evaluated the regucalcin expression in 3 tissues of normal pancreas, especially at the site of exocrine glandular cells and 11 tissues of adenocarcinoma in the pancreas. The dataset of two antibodies (HPA029102 and HPA029103) for regucalcin were used in this analysis.

Pancreatic cancer cells. We used human pancreatic cancer MIA PaCa-2 cells, which possess resistance to drugs and radiation in pancreatic cancer therapy $(8,10)$. This cell line was mutated for the ras gene, but not the rgn gene. Cloned human pancreatic cancer MIA PaCa-2 cell lines [ATCC; CRM-CRL1420, epithelial cell (K-ras Crm)] were obtained from the American Type Culture Collection (ATCC; Rockville, MD, USA).

Regucalcin transfectants. Transfectants, which are overexpressing regucalcin in cloned human pancreatic cancer MIA $\mathrm{PaCa}-2$ cells, were generated in this experiment. The cDNA encoding human regucalcin with full length (900 bp), deleted exon 4 (684 bp), and deleted exon 4 and 5 (552 bp) was isolated and cloned into the pBluescript vector (23). The regucalcin cDNA contains PstI site and an EcoRI fragment (containing the complete coding cDNA) was cloned into the EcoRI site of the pCXN2 expression vector (23). The resultant plasmid was designated as regucalcin/pCXN2 (23). For transient transfection assay, MIA PaCa-2 cells were grown on 24-well plates to $\sim 70 \%$ confluence. Each of regucalcin [including full length (900 bp), deleted exon 4 (684 bp), and deleted exon 4 and 5 (552 bp)]/pCXN2 and pCXN2 vector alone was transfected into MIA PaCa-2 cells using the synthetic cationic lipid components, a Lipofectamine reagent, according to the manufacture's instructions (Promega, Madison, WI, USA) (23). After overnight transfection, neomycin (500-700 $\mu \mathrm{g} / \mathrm{ml}$ of medium (geneticin G418; Sigma-Aldrich) was added to culture wells for selection and cells were cultured for 3 weeks, and then cells were plated at limiting dilution to isolate transfectants. Multiple surviving clones were isolated, transferred to 35-mm dishes, and grown in the medium without neomycine. Regucalcin was stably expressed in the transfectants (clone 1 and 2); protein levels of regucalcin in the transfectants were increased 12.6- or 19.7-fold as compared with that of wild-type cells, respectively. Transfectant of clone 2 was used in this experiment.

Western blotting. MIA PaCa-2 (wild-type) cells, which were transfected with control vector or regucalcin cDNAs with full 
length, deleted exon 4 and deleted exon 4 and 5 were plated in $35-\mathrm{mm}$ dishes at a density of $1 \times 10^{6}$ cells/well in $2 \mathrm{ml}$ of medium, and were cultured in DMEM containing 10\% FBS and $1 \% \mathrm{P} / \mathrm{S}$ for 3 days. Cells were washed twice with iced PBS and removed from the dish with a cell scraper after cell lysis buffer containing protein inhibitors. Removed cells were centrifuged and the pellet was homogenized by sonication in $0.1 \mathrm{ml}$ of iced cell lyses buffer containing protein inhibitors. The homogenate was centrifuged for $10 \mathrm{~min}$ at $17,000 \mathrm{x} \mathrm{g}$, and the protein concentration of supernatant was determined using bovine serum albumin as a standard. Samples $(30 \mu \mathrm{g})$, per lane, of supernatant protein were separated by SDS-PAGE and transferred to nylon membranes for western blotting using antibodies against regucalcin (including 33, 25 and $20 \mathrm{kDa}$ ) $(22,23)$ and other proteins (Cell Signaling Technology, Beverly, MA, USA). Loading controls consisted of $\beta$-actin for the sample proteins. A minimum of 3 blots from independent experiments were scanned on an Epson Perfection 1660 Photo scanner, and bands were quantified using ImageJ. Data from independent experiments were normalized to a percentage of control before averaging as required.

Cell proliferation. Wild-type MIA PaCa- 2 cells ( $1 \times 10^{5} / \mathrm{ml} /$ well) and MIA PaCa- 2 cells $\left(1 \times 10^{5} / \mathrm{ml} /\right.$ well $)$ transfected with regucalcin cDNAs of either full length, deleted exon 4 or deleted exon 4 and 5 were cultured using a 24-well plate in DMEM containing $10 \% \mathrm{FBS}$ and $1 \% \mathrm{P} / \mathrm{S}$ for $1,2,3$ or 7 days in a water-saturated atmosphere containing $5 \% \mathrm{CO}_{2}$ and $95 \%$ air at $37^{\circ} \mathrm{C}(29,30)$. In separate experiments, wild-type MIA PaCa-2 cells $\left(1 \times 10^{5} / \mathrm{ml} /\right.$ well $)$ or transfectants, which were transfected with the regucalcin cDNA of full length, were cultured in DMEM containing $10 \% \mathrm{FBS}$ and $1 \% \mathrm{P} / \mathrm{S}$ in the presence of sodium butyrate (10 and $100 \mu \mathrm{M})$, roscovitine (10 and $100 \mathrm{nM})$, sulforaphane (1 and $10 \mathrm{nM})$, dibucaine $(0.1$ or $1 \mu \mathrm{M})$, Bay $\mathrm{K} 8644(0.1$ or $1 \mu \mathrm{M})$, PD98059 $(1$ or $10 \mu \mathrm{M})$, worthomannin $(0.1$ or $1 \mu \mathrm{M})$, DRB $(0.1$ or $1 \mu \mathrm{M})$, or gemcitabine $(50$ or $100 \mathrm{nM}$ ) for 3 days. After culture, the cells were detached from each culture dish and counted.

Cell death. Wild-type MIA PaCa- 2 cells (1x10 $/ \mathrm{ml} /$ well) and MIA PaCa- 2 cells $\left(1 \times 10^{5} / \mathrm{ml} /\right.$ well) transfected with regucalcin cDNA of either full length, deleted exon 4 or deleted exon 4 and 5. MIA PaCa-2 cells were cultured using a 24-well plate in DMEM containing $10 \% \mathrm{FBS}$ and $1 \% \mathrm{P} / \mathrm{S}$ for 4 days when confluence was reached, and then the cells were additionally cultured for 3 days in the presence or absence of LPS (0.1 or $1 \mu \mathrm{g} / \mathrm{ml})$, TNF- $\alpha(0.1$ or $1 \mathrm{ng} / \mathrm{ml}$ ) (31). In additional experiments, MIA PaCa- 2 cells (wild-type or transfectants; $1 \times 10^{5} / \mathrm{ml} /$ well) were cultured in the presence or absence of LPS $(1 \mu \mathrm{g} / \mathrm{ml})$ or TNF- $\alpha(1 \mathrm{ng} / \mathrm{ml})$ with or without caspase-3 inhibitor $(10 \mu \mathrm{M})$ for $24 \mathrm{~h}$ (31). After culture, cells were detached from each culture dish.

Cell counting. After trypsinization of each culture dish using $0.2 \%$ trypsin plus $0.02 \%$ EDTA in $\mathrm{Ca}^{2+} / \mathrm{Mg}^{2+}$-free PBS for $2 \mathrm{~min}$ at $37^{\circ} \mathrm{C}$, the detached cells from the dish were collected after centrifugation (29-31). Cells were resuspended on PBS solution and stained with eosin. Cell numbers were counted under a microscope using a hemocytometer plate. For each dish, we recorded the average of two counts. Cell number was shown as the number per well of each plate.
Migration assay. We used in vitro scratch assay method for analysis of cell migration in vitro (32). MIA PaCa- 2 cells $\left(1 \times 10^{5}\right.$ cells/ml of plate) of wild-type and transfectants (with full length of regucalcin) were cultured in DMEM containing $10 \%$ FBS and 1\% P/S for $24 \mathrm{~h}$ using 12-well plates at confluence, and then scratch was created in a cell monolayer, capturing the images at the beginning and at 24 or $48 \mathrm{~h}$ of culture during cell migration. Cells in the plate were fixed in $95 \%$ ethanol (ice cold) and stained with crystal violet (1\% in PBS). After drying overnight, the distance in the plates was imaged, and stained cells in the scratched spaces were counted under a microscope to determine the number of migrated cells.

Statistical analysis. The statistical significance was determined using GraphPad InStat version 3 for Windows XP (GraphPad Software, Inc., La Jolla, CA, USA). Multiple comparisons were performed by one-way analysis of variance (ANOVA) with Tukey-Kramer multiple comparisons post-test for parametric data. Survival curves were constructed by Kaplan-Meier analysis and were compared with the log-rank test. Other data were analyzed with the paired or unpaired Student's $t$-test as performed with IBM SPSS Statistics 18 software (IBM, Chicago, IL, USA; http://www.ibm. com). A P-value of <.05 was considered to indicate a statistically significant result.

\section{Results}

Prolonged survival in pancreatic cancer patients with higher regucalcin gene expression. To understand an involvement of regucalcin in the patients of human pancreatic cancers, we analyzed the expression levels of regucalcin in normal pancreatic tissues and pancreatic ductal adenocarcinoma (PDA) in human subjects. We performed microarray analysis to evaluate the regucalcin expression levels in 36 normal pancreas and 36 PDA patients. Most tissues of PDA had a significant lower expression of regucalcin as compared with that of tissues in normal pancreas (Fig. 1A). Quantitative analysis also showed that the expression of regucalcin in PDA patients was remarkably reduced as compared with that in the tissue of normal pancreas (Fig. 1B). To confirm the reduction of regucalcin protein levels, we checked the expression level of regucalcin in 3 tissues of normal pancreas and 11 PDA through the immunohistochemistry database (The Human Protein Atlas). The results using two antibodies showed that the expression of regucalcin in PDA patients were also suppressed as compared of that in normal pancreas (Fig. 1C). Moreover, we compared the outcome between 11 PDA patients with higher regucalcin expression and 14 PDA patients with lower regucalcin expression. The reduction of regucalcin expression was associated with poor prognosis in PDA patients. Survival in pancreatic cancer patients with increased regucalcin gene expression was prolonged (Fig. 1D). These findings may support the view that the suppression of regucalcin gene expression partly contributes in the development of carcinogenesis in human pancreatic cells.

Generation of regucalcin-overexpressing MIA PaCa-2 cells. The cDNA-encoding human regucalcin with full length (33 kDa), deleted exon $4(25 \mathrm{kDa})$, and deleted exon 4 and $5(20 \mathrm{kDa})$ was cloned into the expression vector pCXN2. 


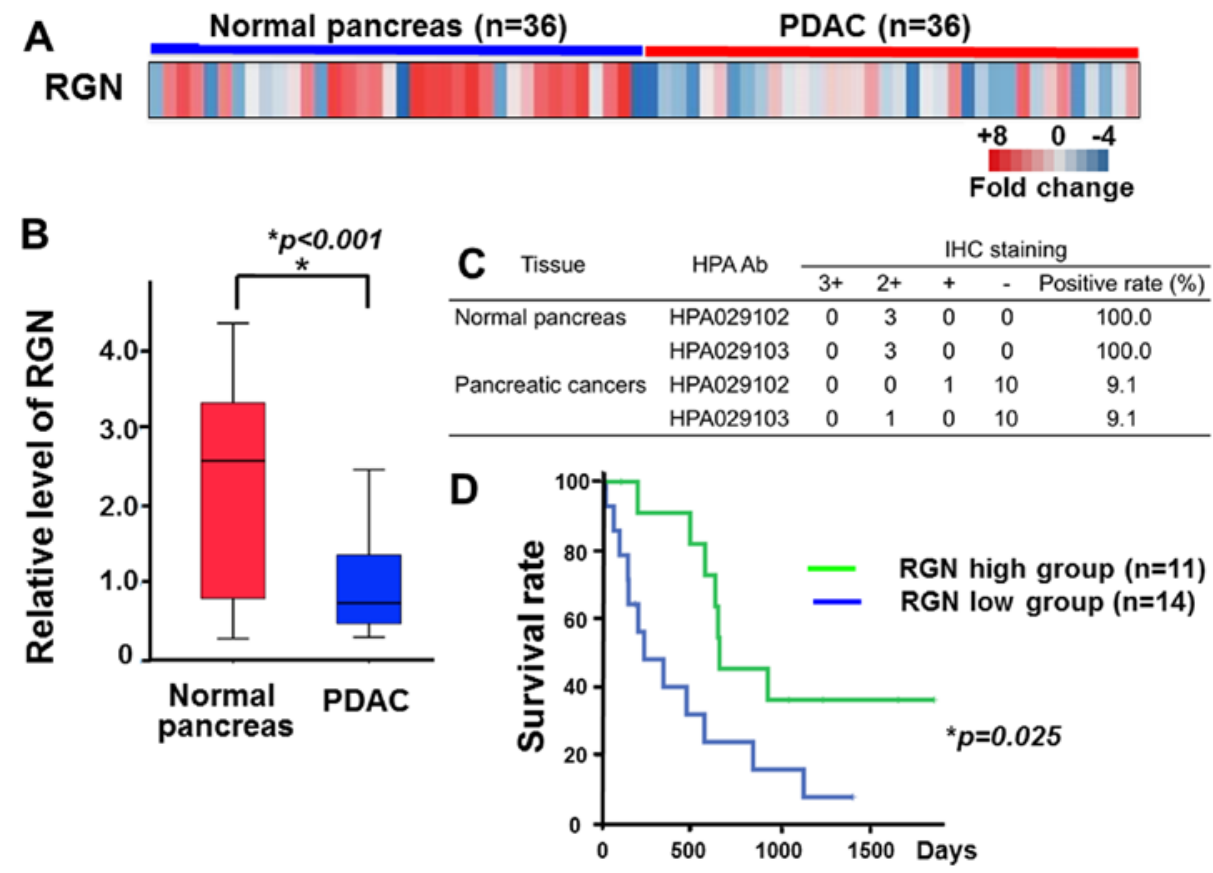

Figure 1. Prolonged survival in pancreatic cancer patients with higher regucalcin gene expression. Reduced regucalcin expression weakens survival of pancreatic cancer patients. (A) Microarray expression analysis of regucalcin in 36 normal pancreas and 36 pancreatic cancers (ref. 25). Each colored square on the bottom right represents the relative mean transcript abundance; highest expression in red, average expression in white, and the lowest expression in blue. (B) Quantification of regucalcin expression in normal pancreas and PDAC similarly to that in (A). Regucalcin expression was suppressed in PDAC patients. (C) The degree of immunohistochemical staining of regucalcin in 3 tissues of normal pancreas and 11 PDAC though immunohistochemistry (IHC) database (The Human Protein Atlas). Regucalcin levels were reduced in PDAC patients. The results of two antibodies are indicated. IHC staining scores: $3+$, strong; $2+$, moderate; +, weak; -, negative. (D) Survival curves for PDAC were in high expression as compared with low expression of regucalcin. PDAC, pancreatic ductal adenocarcinoma; HPA, The Human Protein Atlas; IHC, immunohistochemistry; RGN, regucalcin.

Human pancreatic cancer MIA PaCa-2 cells were generated using the $\mathrm{pCXN} 2$ vector or regucalcin/pCXN2 construct by lipofection. The regucalcin content in the cells transfected with regucalcin cDNA vector of full length $(33 \mathrm{kDa})$ in the clone 1 and 2 was increased 12.6- or 19.7-fold as compared with that of the parental wild-type MIA PaCa-2 cells, respectively (Fig. 2A). We used transfectants of clone 2 in subsequent experiments. Noticeably, the transfectants, which were transfected with deleted exon $4(25 \mathrm{kDa})$ or deleted exon 4 and $5(20 \mathrm{kDa})$, did not express proteins that are associated with 20 or $25 \mathrm{kDa}$ (data not shown). Amino acid structure of both deleted exon 4 and deleted exon 4 and 5 was shown in a previous study (22). Translational process for these proteins was suggested not to function in MIA PaCa-2 cells transfected with the regucalcin cDNA vector with the deleted exon 4 or deleted exon 4 and 5 .

Overexpression of regucalcin suppresses the proliferation in MIA PaCa-2 cells. To determine the effects of the overexpression of endogenous regucalcin on proliferation in human pancreatic cancer MIA PaCa-2 cells in vitro, the cancer cells were cultured for 1, 2, 3 and 7 days. Number of the cells was elevated with increasing the periods of culture (Fig. 2). This increase was suppressed in MIA PaCa- 2 cells overexpressing regucalcin of full length (33 kDa) for 1 (Fig. 2B), 2 (Fig. 2C), 3 (Fig. 2D) and 7 (Fig. 2E) days. We also examined whether cell proliferation is suppressed by the transfection with the regucalcin cDNA [deleted exon $4(25 \mathrm{kDa})$ or deleted exon 4 and $5(20 \mathrm{kDa})]$. In these transfectants, the proliferation was not suppressed by culture for 7 days as compared with that of wild-type cells (Fig. 2B-E). Thus, overexpression of regucalcin with full length was found to specifically possess suppressive effects on the proliferation of MIA PaCa-2 cells in vitro.

Proliferation in MIA PaCa-2 cells was determined in the presence of various inhibitors that induce cell cycle arrest in vitro (Fig. 3). Wild-type cells were cultured for 3 days in the presence of butyrate $(10$ and $100 \mu \mathrm{M})$, roscovitine (10 and $100 \mathrm{nM})$ or sulforaphane $(1$ and $10 \mathrm{nM})(29,33,34)$. The proliferation of wild-type cells was suppressed in the presence of these inhibitors (Fig. 3A). Such effects were not altered in transfectants (Fig. 3B). This result suggested that endogenous regucalcin induces $\mathrm{G} 1$ and $\mathrm{G} 2 / \mathrm{M}$ phase cell cycle arrest in MIA PaCa- 2 cells.

Next, to determine the mechanistic characterization for suppressive effects of regucalcin on cell proliferation, we examined whether suppressive effects of overexpressed exogenous regucalcin on the proliferation in MIA PaCa-2 cells are modulated through various signaling factors that suppress the proliferation. Proliferation in MIA PaCa-2 cells (wild-type) was suppressed in the presence of dibucaine $(0.1$ or $1 \mu \mathrm{M})$, an inhibitor of calcium/calmodulin-dependent protein kinases (29), or Bay $\mathrm{K} 8644(0.1$ or $1 \mu \mathrm{M})$, an agonist of calcium entry into cells (35) (Fig. 4A). Such effects were not seen in transfectants (Fig. 4B). Likewise, proliferation in MIA PaCa-2 cells (wildtype) was suppressed by culture with worthmannin ( 0.1 or $1 \mu \mathrm{M}$ ), an inhibitor of phosphatidylinositol 3-kinase (PI3K) (36), and PD98059 (1 or $10 \mu \mathrm{M})$, an inhibitor of extracellular signal-regulated kinase, ERK, and mitogen-activated protein kinase (MAPK) (37) (Fig. 4C). Suppressive effects of these inhibitors on cell proliferation were not exhibited in the 

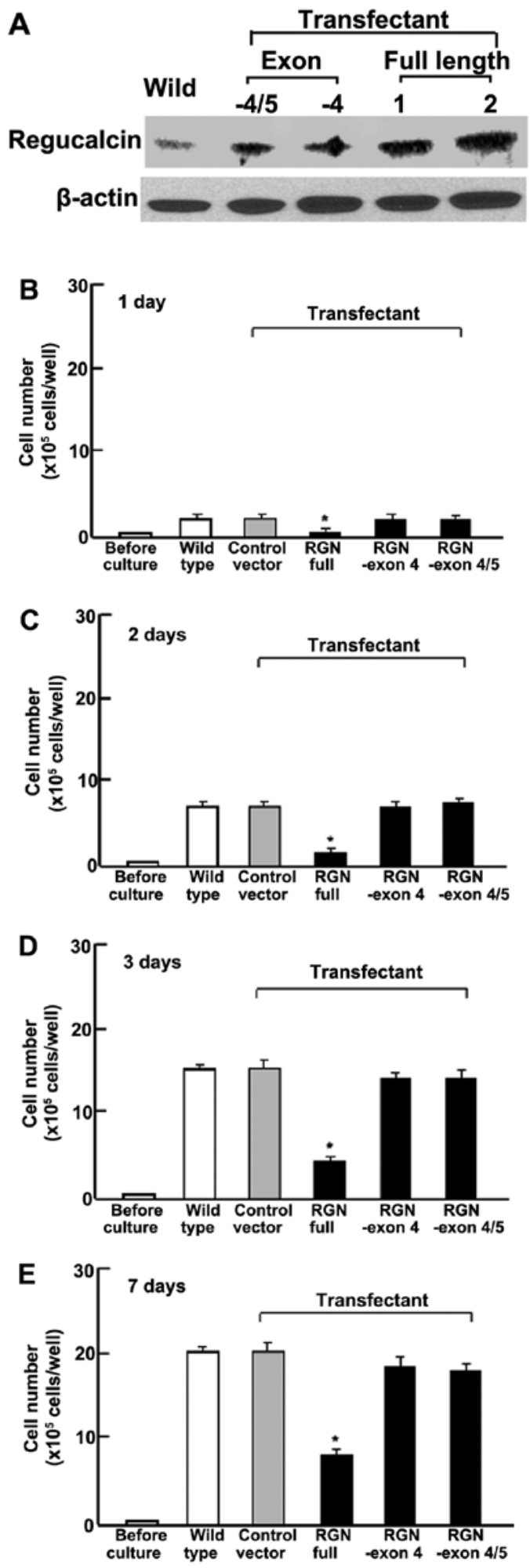

Figure 2. Overexpression of regucalcin (RGN) suppresses the proliferation in human pancreatic cancer MIA PaCa-2 cells. Cells were transfected with regucalcin cDNA vector including full, deleted exon 4 and deleted exon 4 and 5 in vitro. (A) RGN content in the cells cultured for 3 days was analyzed by immunoblotting with an anti-regucalcin antibody. Lane 1, wild-type cells (designated as wild). Lane 2, the cells transfected with the regucalcin cDNA (deleted exon 4 and 5)/pCXN2 (designated as exon 4 and 5). Lane 3, cells transfected with RGN (deleted exon 4)/pCXN2 (designated as exon 4). Lanes 4 and 5, the cells transfected with RGN (full length)/pCXN2 (designated as full RGN clones 1 and 2). These cells were cultured in DMEM for 1 (B), 2 (C), 3 (D) or 7 (E) days. After culture, the number of attached cells on dish was counted. Data are presented as mean \pm SD of 2 replicate wells per data set using different dishes and cell preparations. ${ }^{*} \mathrm{P}<0.001$ vs. wild-type (white bar) or control vector (grey bar). One way ANOVA, Tukey-Kramer post-test.
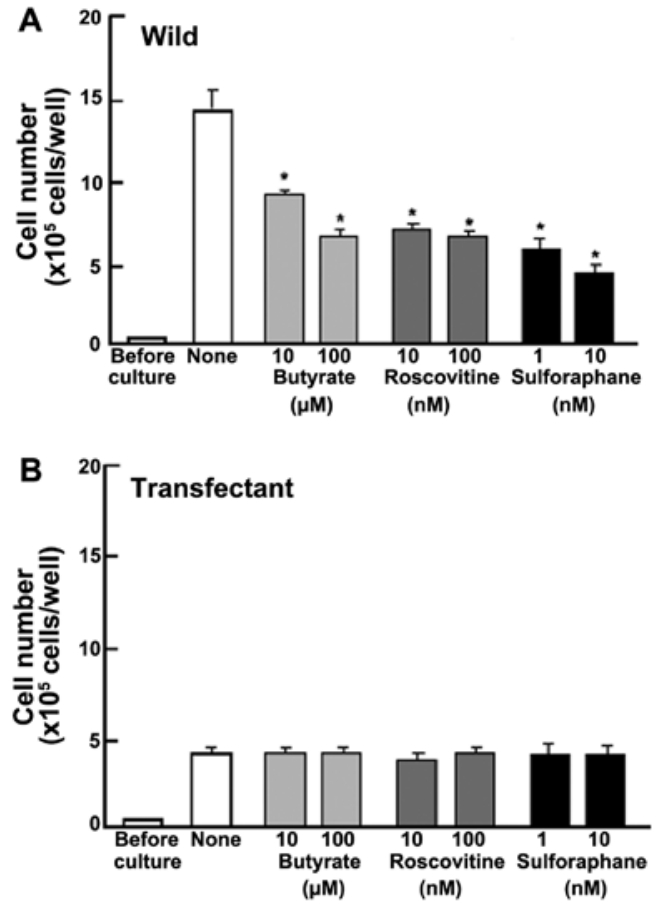

Figure 3. Suppressive effects of regucalcin on the proliferation in human pancreatic cancer MIA PaCa-2 cells are involved in cell cycle arrest by estimating cell cycle inhibitors. (A) Wild-type cells or (B) transfectants overexpressed with regucalcin of full length were cultured for 3 days in the absence or presence of butyrate $(10$ and $100 \mu \mathrm{M})$, roscovitine $(10$ and $100 \mathrm{nM})$ or sulforaphane (1 and $10 \mathrm{nM})$. After culture, the number of attached cells on a dish was counted. Data are presented as mean \pm SD of 2 replicate wells per data set using different dishes and cell preparations. ${ }^{*} \mathrm{P}<0.001$ vs. control (none; white bar). One way ANOVA, Tukey-Kramer post-test.

transfectants (Fig. 4D). DRB is an inhibitor of transcriptional activity with RNA polymerase II inhibition (38). Gemcitabine is a strong antitumor agent that induces nuclear DNA damage (39). Proliferation of MIA PaCa-2 cells was suppressed by culture with DRB $(0.1$ or $1 \mu \mathrm{M})$ or gemcitabine ( 50 or $100 \mathrm{nM}$ ) (Fig. $4 \mathrm{E})$. However, the suppressive effects were not seen with DRB but were seen with gemcitabine in transfectants (Fig. 4F).

Overexpression of regucalcin was found to regulate various protein expressions that are related to signaling pathways in MIA PaCa-2 cells in vitro using western blot analysis (Fig. 5). Protein levels of Akt, phospho-Akt, MAPK, phospho-MAPK, SAPK/JNK, and phospho-SAPK/JNK were decreased by overexpression of regucalcin (Fig. 5A). These results suggested that overexpression of regucalcin suppresses signaling pathways that are related to activation of K-ras in MIA PaCa-2 cells. In addition, overexpression of regucalcin decreased protein levels of K-ras, c-fos and c-jun in MIA PaCa-2 cells (Fig. 5B). Protein levels of $p 53$, a tumor suppressor, were increased by overexpression of regucalcin (Fig. 5B).

Overexpression of regucalcin prevents cell death. To determine the effects of the overexpression of endogenous regucalcin on cell death in MIA PaCa-2 cells, the cells were cultured for 5 days until confluent. Cells at confluence were cultured for an additional $24 \mathrm{~h}$. Number of wild-type cells was decreased in the presence of LPS $(0.1$ or $1 \mu \mathrm{g} / \mathrm{ml})$ or TNF- $\alpha(0.1$ or $1 \mathrm{ng} / \mathrm{ml}$ ), which is known to induce apoptotic cell death (31) (Fig. 6A). Such effects were not seen in the regucalcin (full 

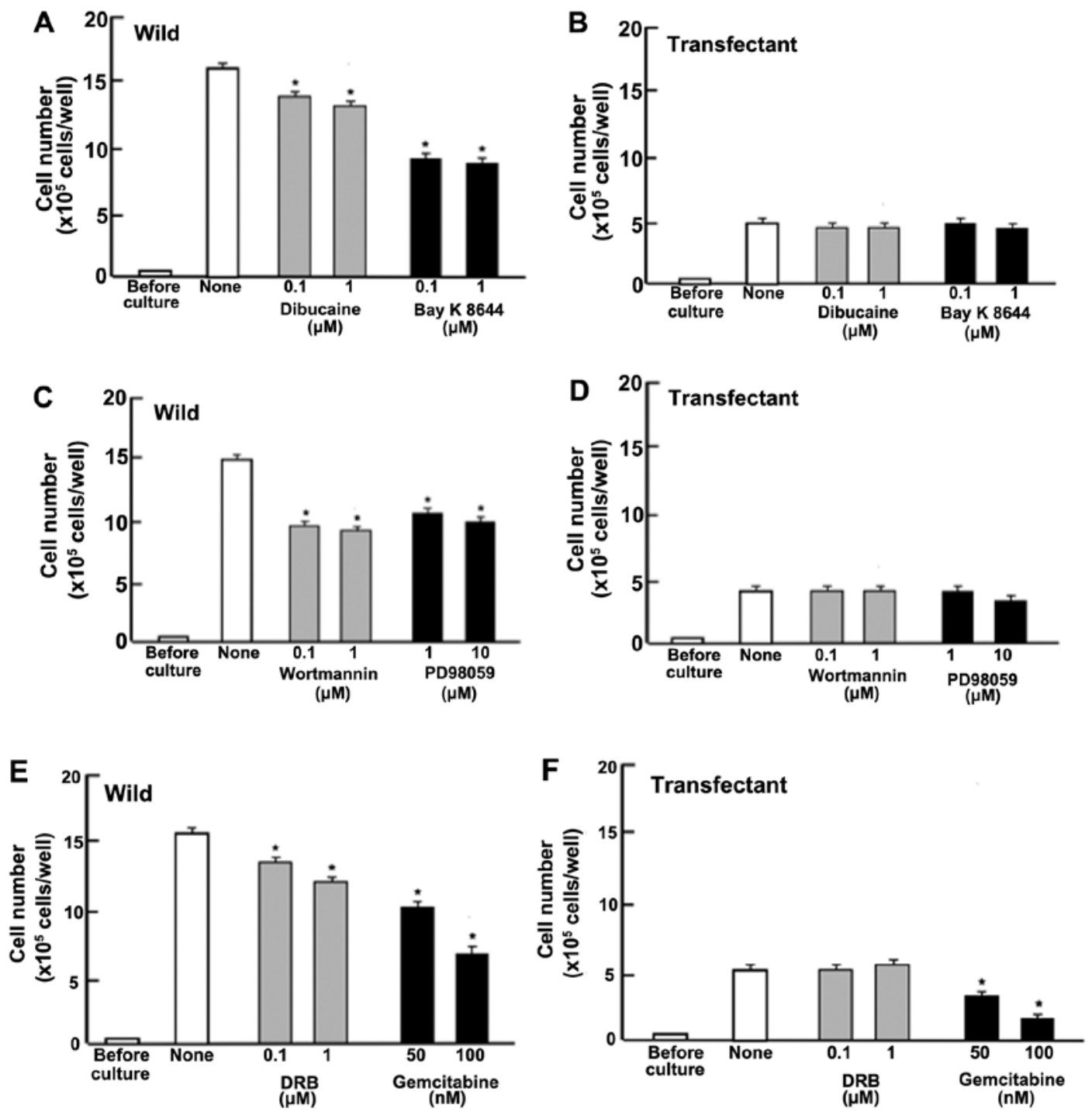

Figure 4. Overexpression of regucalcin regulates various protein levels related to signal transduction in human pancreatic cancer MIA PaCa-2 cells in vitro. Wild-type cells (A, C and E) or transfectants (B, D and F) overexpressin regucalcin (full length) were cultured for 3 days in DMEM containing 10\% FBS and $1 \% \mathrm{P} / \mathrm{S}$ in the presence or absence of dibucaine $(0.1$ or $1 \mu \mathrm{M})$, Bay K $8644(0.1$ or $1 \mu \mathrm{M})$, wortmannin $(0.1$ or $1 \mu \mathrm{M})$, PD98059 $(1$ or $10 \mu \mathrm{M})$, DRB $(0.1$ or $1 \mu \mathrm{M})$ or gemcitabine $(50$ or $100 \mathrm{nM})$. After culture, the number of attached cells on the dish was counted. Data are presented as data as mean $\pm \mathrm{SD}$ of 2 replicate wells per data set using different dishes and cell preparation. $\mathrm{p}<0.001$ vs. control (none; white bar); one-way ANOVA, Tukey-Kramer post-hoc test.

A

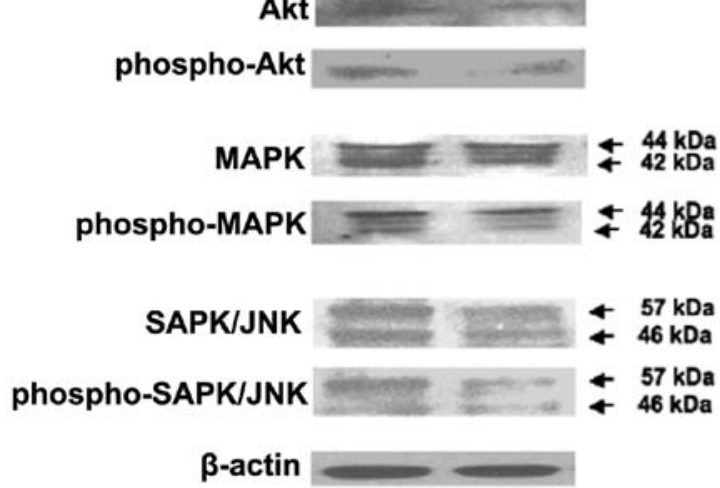

B

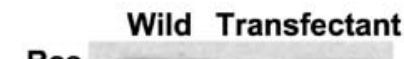

Ras

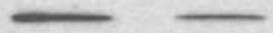

c-fos

c-jun

p-53

$\beta$-actin

Figure 5. Overexpression of regucalcin regulates various protein levels related to signal transduction in human pancreatic cancer MIA PaCa-2 cells in vitro. Wild-type cells or transfectants overexpressing regucalcin (full length) were cultured in DMEM containing 10\% FBS and 1\% P/S for 3 days. After culture, the cells were removed from the dish with a cell scraper, and cell lysis buffer containing protein inhibitors. Part A shows the changes in the protein levels of Akt, MAPK, and SAPK/JNK. Part B indicates the changes in the protein levels of Ras, c-fos, c-jun, and p-53. Samples (30 $\mu \mathrm{g})$ of supernatant protein per lane were separated by SDS-PAGE and transferred to nylon membranes for western blotting using antibodies against various proteins. Data are presented as data set using the cell preparation obtained from different dishes with replicate. 

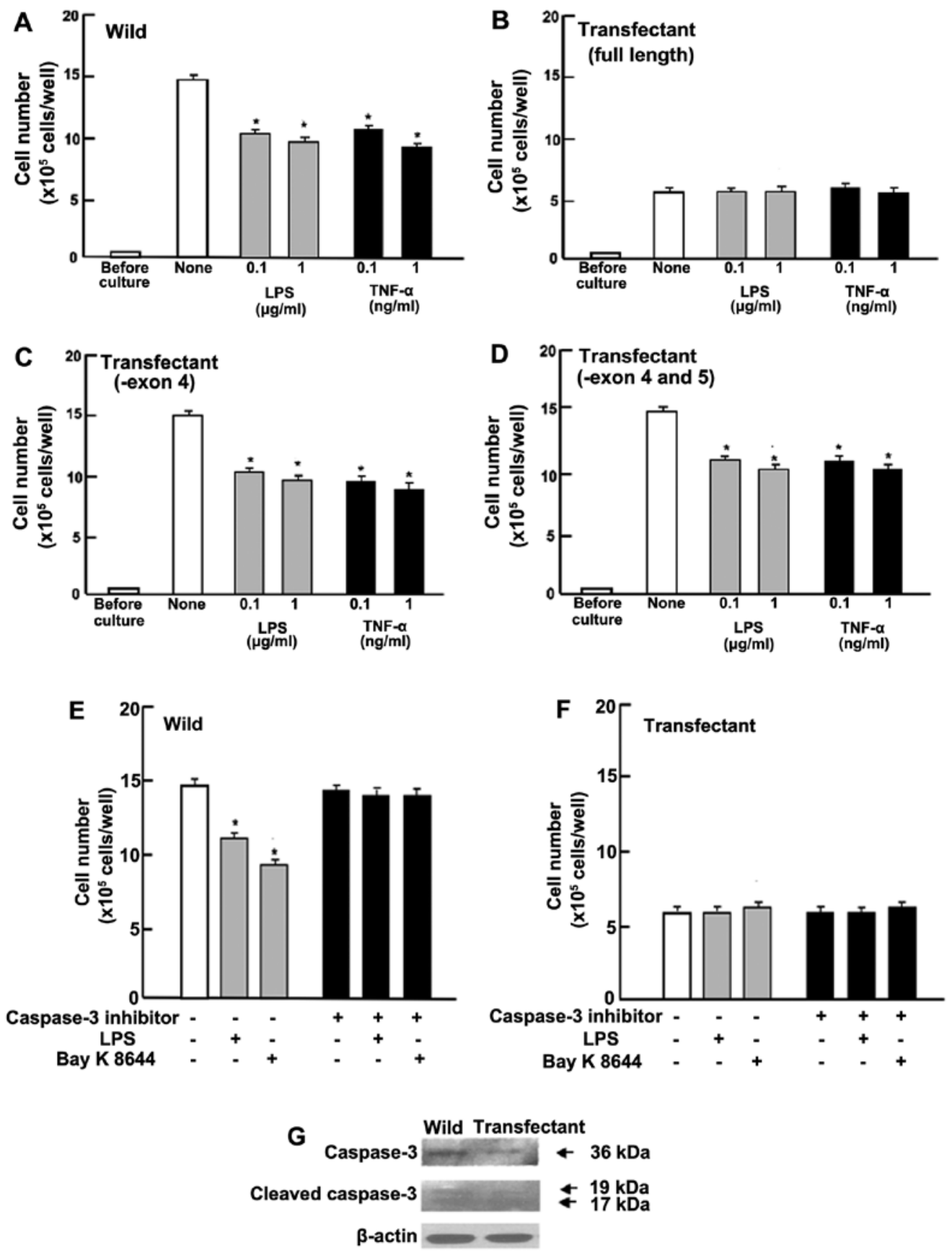

Figure 6. Overexpression of regucalcin prevents cell death induced by stimulation of LPS, TNF- $\alpha$ or Bay K8644 in human pancreatic cancer MIA PaCa-2 cells in vitro. (A) Wild-type cells or (B) transfectants overexpressed regucalcin of full length, (C) deleted exon 4 or (D) deleted exon 4 and 5 were cultured for 5 days until confluence, and then the cells were cultured for an additional $24 \mathrm{~h}$ in the presence of LPS $(0.1 \mathrm{or} 1 \mu \mathrm{g} / \mathrm{ml})$ or TNF- $\alpha(0.1 \mathrm{or} 1 \mathrm{ng} / \mathrm{ml})$. Moreover, (E) wild-type cells or (F) transfectants were cultured for an additional $24 \mathrm{~h}$ in the presence of LPS (1 $\mu \mathrm{M})$ or Bay K8644 (1 $\mu \mathrm{M})$ with or without caspase-3 inhibitor $(10 \mu \mathrm{M})$. After culture, the number of attached cells on dish was counted. (G) The protein levels of caspase-3 and cleavaged caspase-3 with western blotting using the lyses obtained from wild-type cells and transfectants (full length of regucalcin), were cultured for $72 \mathrm{~h}$. Data are presented as mean $\pm \mathrm{SD}$ of 2 replicate wells per data set using different dishes and cell preparations. ${ }^{*} \mathrm{P}<0.001$ vs. control (none; white bar). One way ANOVA, Tukey-Kramer post-test.

length)-overexpressing transfectants that did not exhibit a significant effect on the death in MIA PaCa-2 cells (Fig. 6B). However, the stimulatory effects of LPS $(0.1$ or $1 \mu \mathrm{g} / \mathrm{ml})$ or TNF- $\alpha(0.1$ or $1 \mathrm{ng} / \mathrm{ml})$ on apoptotic death were seen in MIA $\mathrm{PaCa}-2$ cells transfected with the regucalcin cDNA deleted with the exon 4 or with the exon 4 and 5 (Fig. 6C and D). Thus, overexpression of regucalcin with full length was found to specifically prevent the death induced by LPS or TNF- $\alpha$ in MIA PaCa- 2 cells.
Moreover, to determine whether the preventive effects of regucalcin on cell death involved caspase-3, wild-type cells and transfectants (with full length of regucalcin) at confluence after culture for 5 days, were additionally cultured in the presence of LPS $(1 \mu \mathrm{g} / \mathrm{ml})$ or Bay K8644 $(1 \mu \mathrm{M})$ with or without caspase-3 inhibitors $(10 \mu \mathrm{M})$ for $24 \mathrm{~h}$ (Fig. $6 \mathrm{E}$ and $\mathrm{F}$ ). Stimulatory effects of LPS or Bay K8644 on cell death were completely prevented in the presence of caspase- 3 inhibitor (Fig. 6E). LPS- or Bay K8644-induced cell death was not 


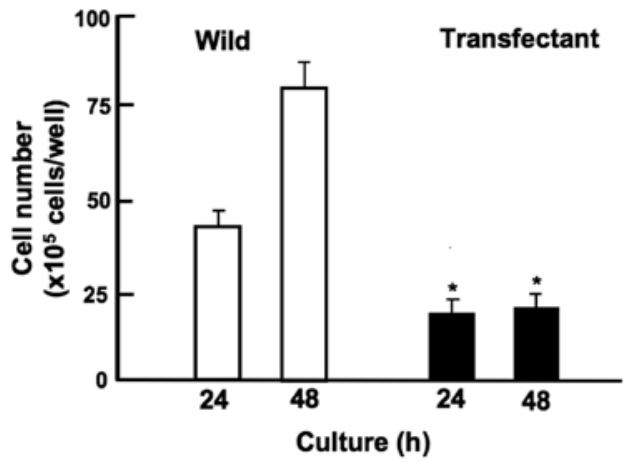

Figure 7. Overexpression of regucalcin suppresses migration of human pancreatic cancer MIA PaCa-2 cells in vitro. MIA PaCa- 2 cells of wild-type and transfectants (full length of regucalcin) were cultured for $24 \mathrm{~h}$ using 12 -well plates until subconfluence, then a scratch was created in a cell monolayer, capturing the images at 24 -h culture during cell migration. Cells migrated into the space of the scratch were fixed in ethanol and stained with crystal violet. Migrated cells were counted under a microscope. Data are presented as mean $\pm \mathrm{SD}$ of 2 replicate wells per data set using different dishes and cell preparation. ${ }^{*} \mathrm{P}<0.001$ vs. control (none; white bar). One way ANOVA, Tukey-Kramer post-test.

seen in the transfectants that were cultured with or without caspase-3 inhibitor (Fig. 6F). In addition, the protein levels of caspase- 3 and cleaved caspase-3 in MIA PaCa- 2 cells were decreased in transfectants with overexpression of regucalcin (full length) (Fig. 6G). These results suggest that endogenous regucalcin prevents cell death due to decreasing the activity of caspase-3 that activates nuclear DNA fragmentation, which induces apoptotic cell death.

Overexpression of regucalcin suppresses cell migration. Overexpression of regucalcin suppressed migration of human pancreatic cancer MIA PaCa-2 cells in vitro (Fig. 7). MIA $\mathrm{PaCa}-2$ cells of wild-type and transfectants (with full length of regucalcin) were cultured for $24 \mathrm{~h}$ using 12-well plates when subconfluent, a scratch was created in a cell monolayer. Cells migrated into the space of the scratch was stained, and counted. Overexpression of regucalcin suppressed migration of cells into the scratched space as compared with that of wildtype cells (Fig. 7).

\section{Discussion}

It was investigated whether or not the regucalcin gene expression is decreased in human pancreatic cancer tissues as compared with that in normal tissues. To understand the involvement of regucalcin in pancreatic cancers, we analyzed the expression level of regucalcin in normal pancreatic tissues and pancreatic ductal adenocarcinoma (PDA). We performed microarray analysis to evaluate the regucalcin expression level in 36 normal pancreas and 36 PDA. Most tissues of PDA had lower expression of regucalcin than that of tissues in normal pancreas. The expression of regucalcin protein was also suppressed in PDA compared to normal pancreas. Moreover, we compared the outcome between 11 PDA with high regucalcin expression and 14 PDA with low regucalcin expression. The reduction of regucalcin expression might be associated with poor prognosis in PDA. Survival in pancreatic cancer patients with the higher regucalcin gene expression was prolonged. These results suggest that the suppression of regucalcin partly contributes the development of carcinogenesis in human pancreatic cells. Overexpression of regucalcin may play a role in the prevention and therapy of human pancreatic cancer.

Moreover, overexpression of regucalcin with full length was found to exhibit anticancer effects in human pancreatic cancer MIA PaCa-2 cells in vitro. Overexpression of regucalcin suppressed the proliferation mediated through various signaling pathways, which stimulate complicated signaling pathways, in human pancreatic cancer MIA PaCa-2 cells in vitro. This effect of regucalcin was independent of cell death. In addition, overexpression of regucalcin was demonstrated to suppress the migration of MIA PaCa-2 cells using scratch assay in vitro, suggesting an anticancer effect in vitro. Higher expression of regucalcin may lead to change in the phenotype revealed in MIA PaCa- 2 cells.

Alternatively spliced variants with the deleted exon 4 $(25 \mathrm{kDa})$ and deleted exon 4 and $5(20 \mathrm{kDa})$ of the regucalcin cDNA has been shown to be present in various types of human cells and tissues, although their protein levels were very low (22). In the present study, we generated the transfectants, which deleted exon 4 or exon both 4 and 5 of the regucalcin cDNA. We did not find any changes in the expression levels of these proteins, and that the proliferation and cell death in these transfectants was not altered as compared with those of wild-type cells and mock-type MIA PaCa- 2 cells. Overexpression of regucalcin with full length was found to specifically exhibit suppressive effects on the proliferation and death in MIA PaCa-2 in vitro.

Suppressive effects of regucalcin overexpression on the proliferation of MIA PaCa-2 cells were not altered in the presence of butyrate, roscovitine or sulforaphane that induce cell cycle arrest. Roscovitine is a potent and selective inhibitor of the cyclin-dependent kinase cdc2, cdk2m and cdk5 (33). Sulforaphane induces G2/M phase cell cycle arrest (34). Butyrate induces an inhibition of G1 progression (25). Endogenous regucalcin was suggested to induce G1 and G2/M phase cell cycle arrest in MIA PaCa-2 cells. This was also confirmed in cloned rat hepatoma H4-II-E cells (25) and cloned normal rat kidney proximal tubular epithelial NRK52E cells (26).

To determine a possible mechanistic characterization of the suppressive effects of regucalcin overexpression on cell proliferation, we used various factors that regulate intracellular signaling pathways. Suppressive effects of regucalcin overexpression on the proliferation in MIA PaCa- 2 cells were not altered in the presence of TNF- $\alpha$, an enhancer of NF- $\kappa B$ signaling (40), Bay K8644, an agonist of $\mathrm{Ca}^{2+}$ entry in cells (35), PD98059, an inhibitor of ERK/MAP kinase-related to signaling pathway (37) or worthmannin, an inhibitor of PI3/ Akt signaling pathway (36). Endogenous regucalcin was suggested to exhibit suppressive effects on the proliferation by inhibiting various intracellular signaling pathways, which are related to $\mathrm{Ca}^{2+}$, PI3/Akt, ERK/MAPK and NF- $\kappa \mathrm{B}$ in MIA PaCa-2 cells. Results with western blotting confirmed that the overexpression of regucalcin led to decreased levels of proteins that were involved in the signaling of Akt and MAPK. Thus, overexpression of regucalcin was demonstrated 
to suppress signaling pathways related to activation of K-ras mutated in MIA PaCa-2 cells.

Noticeably, suppressive effects of regucalcin overexpression on cell proliferation were not changed in the presence of DRB, an inhibitor of transcriptional activity with RNA polymerase II inhibition (38). Endogenous regucalcin was suggested to suppress transcriptional activity in the nucleus of MIA PaCa- 2 cells. Regucalcin has been shown to bind to DNA and regulates nuclear gene expression $(18,41)$. Overexpression of regucalcin has been demonstrated to increase the gene expressions of $p 53$ and $R b$, a tumor suppressor, and to suppress the gene expressions of H-ras, c-jun and c-src, an oncogene, in cloned rat hepatoma H4II-E cells in vitro (41). Overexpression of regucalcin was found to increase the protein levels of $p-53$ and to decrease the protein levels of K-ras, c-jun and c-fos in human pancreatic cancer MIA PaCa- 2 cells in vitro. These results may support the view that regucalcin partly mediates an anticancer effect on the proliferation of MIA PaCa-2 cells by regulating the expression of tumor suppressor proteins and oncogenes.

Overexpression of regucalcin was found to prevent cell death induced by various stimulatory factors including TNF- $\alpha$, LPS and Bay K8644 in MIA PaCa- 2 cells in vitro. These results supported the view that the suppressive effects of regucalcin on the proliferation were not based on cell death. Preventive effect of regucalcin on cell death was not enhanced in the presence of caspase-3 inhibitor. Moreover, overexpression of regucalcin decreased the protein levels of caspase-3 and cleavaged caspase- 3 in MIA PaCa- 2 cells. Endogenous regucalcin was suggested to prevent cell death through the mechanism by which it decreases the activity of caspase-3 that activates nuclear DNA fragmentation that induces apoptosis of cells. It is possible that endogenous regucalcin directly inhibits caspase-3. Regucalcin has also been shown to directly inhibit calcium-activated endonuclease in isolated rat liver nucleus in vitro (42) and to suppress nitric oxide synthetase activity and to regulate the gene expression of various proteins that are involved in apoptosis in cloned rat hepatoma H4-II-E cells in vitro $(20,42)$.

Gemcitabine is well known as an antitumor agent that induces nuclear DNA damage, and it is used clinically for the therapy of human pancreatic cancer (39). This agent suppresses cell proliferation and stimulates apoptotic cell death in various types of cancer cells. Suppressive effects of regucalcin overexpression on the proliferation were potentiated in the presence of gemcitabine in MIA PaCa- 2 cells, suggesting that endogenous regucalcin partly acts in pathways that differ from the action mode of gemcitabine. Regucalcin has been shown to inhibit DNA and RNA synthesis in isolated rat liver nuclei $(18,23,41)$.

In conclusion, the present study demonstrates that increased regucalcin gene expression greatly contributes to prolonged survival in human pancreatic cancer patients. Morover, overexpression of regucalcin was found to suppress the proliferation, which is enhanced through various signaling pathways in human pancreatic cancer MIA PaCa-2 cells in vitro. These findings may support the view that endogenous regucalcin plays a potential role as a suppressor protein in the development of human pancreatic cancer. The regucalcin gene may be a new useful tool in the prevention and therapy in human pancreatic cancer in vivo.

\section{References}

1. Hidalgo M: Pancreatic cancer. N Engl J Med 362: 1605-1617, 2010.

2. Sousa CM and Kimmelman AC: The complex landscape of pancreatic cancer metabolism. Carcinogenesis 35: 1441-1450, 2014.

3. Singh D, Upadhyay G, Srivastava RK and Shankar S: Recent advances in pancreatic cancer: Biology, treatment, and prevention. Biochim Biophys Acta 1856: 13-27, 2015.

4. Zhu YY and Yuan Z: Pancreatic cancer stem cells. Am J Cancer Res 5: 894-906, 2015.

5. Oettle H: Progress in the knowledge and treatment of advanced pancreatic cancer: From benchside to bedside. Cancer Treat Rev 40: 1039-1047, 2014.

6. Moniri MR, Dai LJ and Warnock GL: The challenge of pancreatic cancer therapy and novel treatment strategy using engineered mesenchymal stem cells. Cancer Gene Ther 21: 12-23, 2014.

7. McCarroll JA, Naim S, Sharbeen G, Russia N, Lee J, Kavallaris M, Goldstein D and Phillips PA: Role of pancreatic stellate cells in chemoresistance in pancreatic cancer. Front Physiol 5: 141, 2014.

8. Collins MA and Pasca di Magliano M: Kras as a key oncogene and therapeutic target in pancreatic cancer. Front Physiol 4: 407, 2014.

9. Almoguera C, Shibata D, Forrester K, Martin J, Arnheim N and Perucho M: Most human carcinomas of the exocrine pancreas contain mutant c-K-ras genes. Cell 53: 549-554, 1988.

10. Pylayeva-Gupta Y, Grabocka E and Bar-Sagi D: RAS oncogenes: Weaving a tumorigenic web. Nat Rev Cancer 11: 761-774, 2011.

11. Shimokawa N and Yamaguchi M: Molecular cloning and sequencing of the cDNA coding for a calcium-binding protein regucalcin from rat liver. FEBS Lett 327: 251-255, 1993.

12. Shimokawa N, Matsuda Y and Yamaguchi M: Genomic cloning and chromosomal assignment of rat regucalcin gene. Mol Cell Biochem 151: 157-163, 1995.

13. Thiselton DL, McDowall J, Brandau O, Ramser J, d'Esposito F, Bhattacharya SS, Ross MT, Hardcastle AJ and Meindl A: An integrated, functionally annotated gene map of the DXS8026-ELK1 interval on human Xp11.3-Xp11.23: Potential hotspot for neurogenetic disorders. Genomics 79: 560-572, 2002.

14. Yamaguchi M: A novel $\mathrm{Ca}^{2+}$-binding protein regucalcin and calcium inhibition. Regulatory role in liver cell function. In: Calcium Inhibition. K Kohama (ed). Japan Sci Soc Press, Tokyo and CRC Press, Boca Raton, pp19-41, 1992.

15. Yamaguchi M: Role of regucalcin in maintaining cell homeostasis and function (Review). Int J Mol Med 15: 371-389, 2005.

16. Yamaguchi M: Regucalcin and cell regulation: Role as a suppressor in cell signaling. Mol Cell Biochem 353: 101-137, 2011.

17. Yamaguchi M: The transcriptional regulation of regucalcin gene expression. Mol Cell Biochem 346: 147-171, 2011.

18. Yamaguchi M: Role of regucalcin in cell nuclear regulation: Involvement as a transcription factor. Cell Tissue Res 354: 331-341, 2013.

19. Yamaguchi M: Suppressive role of regucalcin in liver cell proliferation: Involvement in carcinogenesis. Cell Prolif 46: 243-253, 2013.

20. Yamaguchi M: The anti-apoptotic effect of regucalcin is mediated through multisignaling pathways. Apoptosis 18: 114-1153, 2013.

21. Yamaguchi M: Involvement of regucalcin as a suppressor protein in human carcinogenesis: Insight into the gene therapy. J Cancer Res Clin Oncol 141: 1333-1341, 2015.

22. Murata T and Yamaguchi M: Alternatively spliced variants of the regucalcin gene in various human normal and tumor tissues. Int J Mol Med 34: 1141-1146, 2014.

23. Misawa H, Inagaki S and Yamaguchi M: Suppression of cell proliferation and deoxyribonucleic acid synthesis in the cloned rat hepatoma H4-II-E cells overexpressing regucalcin. J Cell Biochem 84: 143-149, 2001.

24. Yamaguchi M and Murata T: Suppressive effects of exogenous regucalcin on the proliferation of human pancreatic cancer MIA PaCa-2 cells in vitro. Int J Mol Med 35: 1773-1778, 2015.

25. Badea L, Herlea V, Dima SO, Dumitrascu T and Popescu I: Combined gene expression analysis of whole-tissue and microdissected pancreatic ductal adenocarcinoma identifies genes specifically overexpressed in tumor epithelia. Hepatogastroenterology 55: 2016-2027, 2008. 
26. Collisson EA, Sadanandam A, Olson P, Gibb WJ, Truitt M, Gu S, Cooc J, Weinkle J, Kim GE, Jakkula L, et al: Subtypes of pancreatic ductal adenocarcinoma and their differing responses to therapy. Nat Med 17: 500-503, 2011.

27. Uhlen M, Oksvold P, Fagerberg L, Lundberg E, Jonasson K, Forsberg M, Zwahlen M, Kampf C, Wester K, Hober S, et al: Towards a knowledge-based Human Protein Atlas. Nat Biotechnol 28: 1248-1250, 2010.

28. Uhlén M, Fagerberg L, Hallström BM, Lindskog C, Oksvold P, Mardinoglu A, Sivertsson A, Kampf C, Sjöstedt E, Asplund A, et al: Proteomics. Tissue-based map of the human proteome. Science 347: 1260419, 2015.

29. Yamaguchi M and Daimon Y: Overexpression of regucalcin suppresses cell proliferation in cloned rat hepatoma H4-II-E cells: Involvement of intracellular signaling factors and cell cycle-related genes. J Cell Biochem 95: 1169-1177, 2005.

30. Nakagawa T, Sawada N and Yamaguchi M: Overexpression of regucalcin suppresses cell proliferation of cloned normal rat kidney proximal tubular epithelial NRK52E cells. Int J Mol Med 16: 637-643, 2005

31. Izumi $\mathrm{T}$ and Yamaguchi M: Overexpression of regucalcin suppresses cell death in cloned rat hepatoma H4-II-E cells induced by tumor necrosis factor-alpha or thapsigargin. J Cell Biochem 92: 296-306, 2004.

32. Liang CC, Park AY and Guan JL: In vitro scratch assay: A convenient and inexpensive method for analysis of cell migration in vitro. Nat Protoc 2: 329-333, 2007.

33. Meijer L, Borgne A, Mulner O, Chong JP, Blow JJ, Inagaki N, Inagaki M, Delcros JG and Moulinoux JP: Biochemical and cellular effects of roscovitine, a potent and selective inhibitor of the cyclin-dependent kinases cdc2, cdk2 and cdk5. Eur J Biochem 243: 527-536, 1997.

34. Singh SV, Herman-Antosiewicz A, Singh AV, Lew KL, Srivastava SK, Kamath R, Brown KD, Zhang L and Baskaran R: Sulforaphane-induced G2/M phase cell cycle arrest involves checkpoint kinase 2-mediated phosphorylation of cell division cycle 25C. J Biol Chem 279: 25813-25822, 2004.
35. Cano-Abad MF, Villarroya M, García AG, Gabilan NH and López MG: Calcium entry through L-type calcium channels causes mitochondrial disruption and chromaffin cell death. J Biol Chem 276: 39695-39704, 2001

36. Serrano-Nascimento C, da Silva Teixeira S, Nicola JP, Nachbar RT, Masini-Repiso AM and Nunes MT: The acute inhibitory effect of iodide excess on sodium/iodide symporter expression and activity involves the PI3K/Akt signaling pathway. Endocrinology 155: 1145-1156, 2014.

37. Chen S, Wang Y, Ruan W, Wang X and Pan C: Reversing multidrug resistance in hepatocellular carcinoma cells by inhibiting extracellular signal-regulated kinase/mitogen-activated protein kinase signaling pathway activity. Oncol Lett 8: 2333-2339, 2014.

38. Palangat M, Grass JA, Langelier MF, Coulombe B and Landick R: The RPB2 flap loop of human RNA polymerase II is dispensable for transcription initiation and elongation. Mol Cell Biol 31: 3312-3325, 2011.

39. Tang SC and Chen YC: Novel therapeutic targets for pancreatic cancer. World J Gastroenterol 20: 10825-10844, 2014.

40. Li Y, Li A, Strait K, Zhang H, Nanes MS and Weitzmann MN Endogenous TNFalpha lowers maximum peak bone mass and inhibits osteoblastic Smad activation through NF-kappaB. J Bone Miner Res 22: 646-655, 2007.

41. Tsurusaki Y and Yamaguchi M: Role of regucalcin in liver nuclear function: Binding of regucalcin to nuclear protein or DNA and modulation of tumor-related gene expression. Int J Mol Med 14: 277-281, 2004.

42. Yamaguchi M and Sakurai T: Inhibitory effect of calciumbinding protein regucalcin on $\mathrm{Ca}^{2+}$-activated DNA fragmentation in rat liver nuclei. FEBS Lett 279: 281-284, 1991 